\title{
Medium Cut-Off Dialysis Membranes: Can They Have Impact on Outcome of COVID-19 Hemodialysis Patients?
}

\author{
Serkan Feyyaz Yalın ${ }^{a} \quad$ Mehmet Rıza Altıparmak ${ }^{b}$ Mevlut Tamer Dincer ${ }^{b}$ \\ Serap Yadigar ${ }^{\mathrm{a}}$ Ahmet Murt $^{\mathrm{b}}$ Ergun Parmaksiz ${ }^{\mathrm{a}}$ Claudio Ronco ${ }^{\mathrm{c}, \mathrm{d}}$

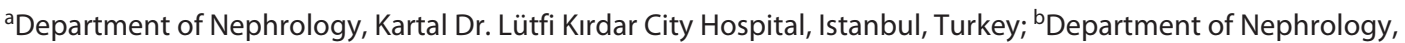 \\ Cerrahpaşa Faculty of Medicine, Istanbul University-Cerrahpaşa, Istanbul, Turkey; 'Department of Medicine (DIMED), \\ University of Padova, Padova, Italy; ${ }^{d}$ Department of Nephrology, Dialysis and Transplantation, San Bortolo Hospital, \\ International Renal Research Institute of Vicenza, Vicenza, Italy
}

Keywords
COVID-19 $\cdot$ Cytokines · Dialysis membranes $\cdot$ Hemodialysis · Outcome

\begin{abstract}
Introduction: Severe acute respiratory syndrome coronavirus-2 may lead to high levels of expression of inflammatory cytokines. Medium cut-off (MCO) membranes may make greater clearances for large-middle molecules (including cytokines) than low-flux (LF) membranes. In this study, we aimed to evaluate the impact of MCO membranes on outcome of COVID-19 patients on hemodialysis (HD). Methods: Sixty COVID-19 HD patients were included in this study. The patients were categorized into 2 groups regarding type of HD membranes. Clinical data were taken from medical records. Results: Initial crp and ferritin levels, which are surragates of cytokine storm and severity of disease in COVID-19, were significantly higher in MCO membrane group compared to LF group ( $p=0.037$ and 0.000 , respectively). Although there were more patients with severe disease in MCO group, there were no significant differences regarding need for intensive care unit and death. Conclusion: It may be an option to use MCO membranes in HD patients with COVID-19 in order to reduce cytokine levels and prevent cytokine storm.
\end{abstract}

(c) 2021 S. Karger AG, Basel

\section{Introduction}

Severe acute respiratory syndrome coronavirus-2 (SARS-CoV-2), which is a novel type of coronavirus, first emerged in December 2019 in Wuhan, China [1]. It has been reported that SARS-CoV may damage the respiratory system and causes serious outbreaks with high mortality rate $[2,3]$. Reports indicate that individuals with older age and/or with underlying illnesses, such as diabetes mellitus, hypertension, or cardiovascular diseases, indicate poor prognosis for COVID-19 [4,5]. These comorbidities are common in hemodialysis (HD) patients. Moreover, patients with chronic kidney failure have impaired innate and adaptive immunity. These result in immunodepression which increases vulnerability to infections [6].

During COVID-19, high levels of expression of inflammatory cytokines may activate the T-helper type 1 (Th1) cell response [7]. Th1 activation is paramount importance of activation of specific immunity [8]. Severity of the disease is well correlated with high serum levels of interleukin-2 receptor and interleukin 6 (IL-6) [9]. IL-6 stimulates production of CRP [10]. In addition, the activation of the monocyte-macrophage system, which is a vital part of inflammatory storm, stimulates ferritin pro-

Serkan Feyyaz Yalın

Department of Nephrology, Cerrahpasa Medical Faculty, Istanbul University Kocamustafapasa Cad. No. 53

Istanbul 34098 (Turkey)

serkanfyalin@yahoo.com 
duction [11]. The cytokine storm causes organ dysfunction, which may cause death [12].

$\mathrm{HD}$ is an extracorporeal process in which waste products that accumulate in patients with ESRD, is removed by a semipermeable membrane [13]. Three types of HD membranes are described: low flux (LF), high flux (HF), and medium cutoff (MCO) [14]. MCO membranes may make greater clearances and intradialytic reduction ratios for large-middle molecules (including cytokines) than LF and HF membranes [15]. In this study, we aimed to evaluate the impact of the type of $\mathrm{HD}$ membranes (MCO membrane vs. LF membrane) on outcome of COVID-19 patients on HD.

\section{Patients and Methods}

\section{Study Setting}

This study was performed in 2 tertiary care university hospitals that are serving in an area with approximately 16 million residents. This study was done in compliance with the Declaration of Helsinki. Institutional approval was taken from the local ethical review committee. The participants' identities were kept confidential.

\section{Study Population}

All HD files of the COVID-19 patients who had chronic HD treatment between March 11, 2020, and September 1, 2020, were retrospectively examined. Sixty COVID-19 HD patients were enrolled in this study from 2 hospitals. One of the hospitals used routine MCO membrane during coronavirus pandemic, and the other 1 used routine LF membrane during that period. Raw clinical profile of the patients was evaluated without randomization.

\section{Data Collection}

The medical data of the patients were retrospectively obtained by a physician who did not know the outcome of the patients. Clinical data, etiologies of kidney diseases, comorbidities, laboratory and radiologic findings, and types of treatments were taken from medical records.

\section{Patient Management}

The positive RT-PCR and/or radiological findings were used to confirm the diagnosis. Disease activity was described as follows: asymptomatic, mild disease (symptoms with or without mild dyspnea), moderate disease (dyspnea requiring oxygen therapy), and severe disease (dyspnea requiring intensive care treatment). All HD patients were hospitalized due to high mortality risk for COVID-19.

\section{Antiviral and Cytokine-Targeted Therapy}

Our managements were based on a national guide written by the scientific board of our country and published by our health ministry [16]. All of the hospitalized patients were initially treated with hydroxychloroquine, azithromycin, and oseltamivir. Then, azithromycin and oseltamivir were removed from initial treatment. Favipiravir was given to refractory cases. Tocilizumab was used in the management of cytokine release syndrome which developed especially in severe cases.
Anticoagulation and Oxygen Treatment

Standard heparin or low-molecular-weight heparin was used in all patients unless contraindicated. Oxygen treatment was given to the patients with oxygen saturation below $92 \%$. If respiratory failure progressed, firstly noninvasive ventilation and then mechanical ventilation was performed in the intensive care unit.

\section{Statistical Analysis}

Data were expressed as mean \pm SD. Quantitative data were compared using either Student's $t$ test or Mann-Whitney U test. Qualitative data were compared using $\chi^{2}$ test. All computations were made using the SPSS for Windows v.17.0 software (SPSS Inc., Chicago, IL, USA). $p$ values of $<0.05$ were considered significant.

\section{Results}

Sixty HD patients were enrolled in this study. Etiologies of kidney disease in group 1 were as follows: hypertension in $8(33.3 \%)$ patients, diabetes mellitus in 5 $(20.8 \%)$, chronic glomerulonephritis in $2(8.3 \%)$, autosomal dominant polycystic kidney disease in $2(8.3 \%)$, collagen vascular diseases in $1(4.2 \%)$, multiple myeloma in 1 (4.2\%), and unknown disease in 5 (20.9\%). Etiologies of kidney disease in group 2 were as follows: diabetes mellitus in $12(33.3 \%)$ patients, hypertension in $10(27.8 \%)$, collagen vascular diseases in 3 (8.4\%), chronic glomerulonephritis in $2(5.5 \%)$, autosomal dominant polycystic kidney disease in 1 (2.8\%) and unknown in 8 (22.2\%).

MCO (Theranova 400, Baxter ${ }^{\mathrm{TM}}$, Deerfield, IL, USA) membranes were used in 24 patients and LF membranes (Elisio-21 M, Nipro ${ }^{\mathrm{TM}}$, Osaka, Japan) were used in 36 patients. Initial demographics and clinical characteristics and laboratory findings of 2 groups are shown in Table 1. Mean age of 2 groups was similar. There were significantly more patients with comorbidities in $\mathrm{MCO}$ group compare to LF group $(p=0.000)$. Serum Cr levels were significantly higher in MCO group compare to LF group $(p=0.000)$. Initial crp and ferritin, which are surragates of cytokine storm in COVID-19, were significantly higher in MCO group compare to LF group ( $p=0.037$ and 0.000 , respectively).

Symptoms and physical findings during onset of diagnosis are shown in online suppl. Table 1; see www. karger.com/doi/10.1159/000513621 for all online suppl. material. Majority of the patients had fever, cough, dyspnea, and myalgia. All of the patients had radiological findings for COVID-19, whereas 25 (50\%) patients had positive PCR test for COVID-19.

Disease activity of the patients during onset of diagnosis is shown in online suppl. Table 2. Regarding LF group, majority of the patients had mild-moderate disease activ- 
Table 1. Demographics and clinical characteristics and initial laboratory findings of 2 groups

\begin{tabular}{lccc}
\hline & $\begin{array}{l}\text { MCO membrane, } \\
n=24\end{array}$ & $\begin{array}{l}\text { LF membrane, } \\
n=36\end{array}$ & $p$ value \\
\hline Age, years & $64.3 \pm 12.9$ & $61.6 \pm 15.6$ & 0.492 \\
Gender (male/female) & $13 / 11$ & $17 / 19$ & 0.598 \\
Dialysis length, month & $33.4 \pm 38.2$ & $48.4 \pm 44.4$ & 0.146 \\
Mean arterial pressure, mm Hg & $95.2 \pm 13.2$ & $95.4 \pm 11.6$ & 0.958 \\
Presence of hypertension, $n(\%)$ & $19(79.1)$ & $29(80.5)$ & 0.895 \\
Presence of diabetes mellitus, $n(\%)$ & $5(20.8)$ & $14(38.8)$ & 0.141 \\
Presence of other comorbidities, ${ }^{\dagger} n(\%)$ & $21(87.5)$ & $10(27.7)$ & $\mathbf{0 . 0 0 0}$ \\
Use of ACE-I/ARB, $n(\%)$ & $5(20.8)$ & $2(5.5)$ & 0.071 \\
Hemoglobin, g/dL & $9.9 \pm 1.4$ & $10.2 \pm 1.6$ & 0.474 \\
WBCs, $10^{3} / \mu \mathrm{L}$ & $8.441 \pm 4.132$ & $7.847 \pm 3.844$ & 0.712 \\
Lymphocyte, $10^{3} / \mu \mathrm{L}$ & $1.033 \pm 0.363$ & $1.060 \pm 0.594$ & 0.826 \\
Platelet count, $10^{3} / \mu \mathrm{L}$ & $237,950 \pm 99,070$ & $202,194 \pm 70,090$ & 0.107 \\
CRP, mg/L & $136 \pm 92.7$ & $91.3 \pm 69.1$ & $\mathbf{0 . 0 3 7}$ \\
Procalcitonin, $\mathrm{ng} / \mathrm{mL}$ & $1 \pm 0.81$ & $6.1 \pm 12.3$ & 0.123 \\
Ferritin, $\mu \mathrm{g} / \mathrm{L}$ & $624.2 \pm 329.8$ & $\mathbf{0 . 0 0 0}$ \\
Serum Cr, $\mathrm{mg} / \mathrm{dL}$ & $3,537.6 \pm 8,544$ & $6.2 \pm 2$ & $\mathbf{0 . 0 0 0}$ \\
Kt/V & $1.33 \pm 2.8$ & $1.25 \pm 0.15$ & 0.09 \\
\hline
\end{tabular}

ACE-İ, angiotensin-converting enzyme inhibitors; ARB, angiotensin II receptor blockers; LF, low flux; MCO, medium cutoff. Bold values represent statistical significance with $p<0.05 .{ }^{\dagger}$ Ischemic heart disease, heart failure, chronic obstructive pulmonary disease, cerebrovascular disease, malignancy, chronic liver disease, and systemic vasculitis.

ity. Whereas, there were more severe patients in $\mathrm{MCO}$ group than LF group in terms of disease activity $(p=$ $0.001)$.

Regarding treatment types, 2 groups were treated with similar drugs according to severity of the disease (online suppl. Table 3). In the MCO group, more patients needed to require favipiravir and tocilizumab treatments compared to LF group.

The outcomes of 2 groups are shown in online suppl. Table 4 . Length of hospital stay was significantly longer in MCO group compared to LF group $(p=0.022)$. However, there were no significant differences between 2 groups in terms of need for intensive care unit and death.

\section{Discussion}

COVID-19 is a life-threatening disease. One of the most important complications of the virus is hemophagocytic lymphohistiocytosis (HLH)-macrophage activation syndrome (MAS) via cytokine storm. Laboratory signs and symptoms of HLH-MAS include severe elevation in acute phase parameters such as CRP and ferritin [17]. HLH-MAS causes organ dysfunction, which may cause death [12].
There is currently no cure for COVID-19. Therefore, clinicians are nowadays trying to treat COVID-19 patients who progressed to HLH-MAS by agents such as antiviral drugs, glucocorticoids, IL-6 antagonists, IL-1 antagonists, intravenous immunoglobulin, convalescent plasma, and janus kinase inhibitors [17]. Also, it was speculated that blood purification technology can prevent COVID-19 patients from severe pneumonia via cytokine clearance [18].

MCO is a novel type membrane for HD. In a study performed by Kim et al. [15], it was shown that HD with MCO membranes achieved significantly greater reduction ratio of large-middle molecules (including cytokines such as IL-1 and IL-6) than HD with conventional membranes and online-hemodiafiltration. In addition, Cozzolino et al. [19] found a reduction in the incidence of infections with MCO membrane compared to HF membrane.

In this study, we evaluated the raw clinical profile of COVID-19 HD patients in 2 hospitals that were using different types of membranes during pandemic. We aimed to see impact of MCO membranes on survival of patients with COVID-19.

Regarding clinical symptoms and laboratory results (especially CRP and ferritin levels), there were more severe COVID-19 patients in MCO group compared to LF group. 
In addition, MCO group patients stayed longer in the hospital due to possible severity of the disease. Despite these data, there were no differences between the 2 groups in terms of need for intensive care unit and death. In light of these results, we can assume that MCO membranes may protect patients from HLH-MAS via cytokine purification.

The limitations of our study are that we performed this study in a small population. In small groups, it may be difficult to interpret statistical analysis. In addition, we could not measure IL-6 levels before and after HD sessions in order to see the reduction in IL-6 level which is a good marker for inflammation in COVID-19 disease.

In conclusion, it may be an option to use MCO membranes in HD patients with COVID-19 in order to reduce cytokine levels and prevent cytokine storm. Also, we can speculate that COVID-19 patients with cytokine storm may benefit from standard HD with MCO membranes regardless of renal failure.

\section{Statement of Ethics}

Subjects have given their written informed consent, and this study protocol was approved by the local committee on human research (IRB no:21.4.2020/A-10).

\section{Conflict of Interest Statement}

In the last 3 years, $C R$ has been consulting or part of advisory boards for ASAHI, Astute, Baxter, Biomerieux, B. Braun, Cytosorbents, ESTOR, FMC, GE, Jafron, Medtronic, and Toray. The other authors have no conflicts of interest to declare.

\section{Funding Sources}

This research received no specific grant from any funding agency.

\section{Author Contributions}

S.F.Y.: conception, design, analysis, revising, and final approval. M.R.A.: conception, analysis, revising, and final approval. M.T.D.: design, analysis, drafting, and revising. S.Y.: acquisition of data, analysis, drafting, and revising. A.M.: design, analysis, drafting, and revising. E.P.: conception, design, analysis, and drafting. C.R.: design, analysis, revising, and final approval.

\section{References}

1 Yang CL, Qiu X, Zeng YK, Jiang M, Fan HR, Zhang ZM. Coronavirus disease 2019: a clinical review. Eur Rev Med Pharmacol Sci. 2020 Apr;24(8):4585-96.

2 Chen N, Zhou M, Dong X, Qu J, Gong F, Han $\mathrm{Y}$, et al. Epidemiological and clinical characteristics of 99 cases of 2019 novel coronavirus pneumonia in Wuhan, China: a descriptive study. Lancet. 2020 Feb 15;395(10223):50713.

3 Pisani A, Rizzo M, Angelucci V, Riccio E. COVID-19 experience in hemodialysis patients: a cue for therapeutic heparin-based strategies? Nephron. 2020;144(8):383-5.

4 Xiong F, Tang H, Liu L, Tu C, Tian JB, Lei CT, et al. Clinical characteristics of and medical interventions for COVID-19 in hemodialysis patients in Wuhan, China. J Am Soc Nephrol. 2020 Jul;31(7):1387-97.

5 Channappanavar R, Perlman S. Pathogenic human coronavirus infections: causes and consequences of cytokine storm and immunopathology. Semin Immunopathol. $2017 \mathrm{Jul}$; 39(5):529-39.

6 Kato S, Chmielewski M, Honda H, PecoitsFilho R, Matsuo S, Yuzawa Y, et al. Aspects of immune dysfunction in end-stage renal disease. Clin J Am Soc Nephrol. 2008 Sep;3(5): 1526-33.
7 Huang C, Wang Y, Li X, Ren L, Zhao J, Hu Y, et al. Clinical features of patients infected with 2019 novel coronavirus in Wuhan, China. Lancet. 2020 Feb 15;395(10223):497-506.

8 Marchingo JM, Sinclair LV, Howden AJ, Cantrell DA. Quantitative analysis of how Myc controls T cell proteomes and metabolic pathways during $\mathrm{T}$ cell activation. Elife. 2020 Feb 5;9:e53725.

9 Chen L, Liu HG, Liu W, Liu J, Liu K, Shang J, et al. [Analysis of clinical features of $29 \mathrm{pa}$ tients with 2019 novel coronavirus pneumonia]. Zhonghua Jie He He Hu Xi Za Zhi. 2020 Mar 12;43(3):203-8.

10 Del Giudice M, Gangestad SW. Rethinking IL-6 and CRP: why they are more than inflammatory biomarkers, and why it matters. Brain Behav Immun. 2018 May;70:61-75.

11 Guo W, Li M, Dong Y, Zhou H, Zhang Z, Tian $\mathrm{C}$, et al. Diabetes is a risk factor for the progression and prognosis of COVID-19. Diabetes Metab Res Rev. 2020 Mar 31;e3319.

12 Song P, Li W, Xie J, Hou Y, You C. Cytokine storm induced by SARS-CoV-2. Clin Chim Acta. 2020 Oct;509:280-7.

13 Ronco C, Clark WR. Haemodialysis membranes. Nat Rev Nephrol. 2018 Jun;14(6):394-410.
14 Zweigart C, Boschetti-de-Fierro A, Hulko M, Nilsson LG, Beck W, Storr M, et al. Medium cut-off membranes: closer to the natural kidney removal function. Int J Artif Organs. 2017 Jul 5;40(7):328-34.

15 Kim TH, Kim SH, Kim TY, Park HY, Jung KS Lee $\mathrm{MH}$, et al. Removal of large middle molecules via haemodialysis with medium cut-off membranes at lower blood flow rates: an observational prospective study. BMC Nephrol. 2019 Dec 31;21(1):2.

16 Turkey_SARSCoV2InfectionGuide. Available from: https://www.fip.org/files/content/ priority-areas/coronavirus/mo-resources/ Turkey_SARSCoV2InfectionGuide.pdf.

17 Halacli B, Topeli A. Treatment of the cytokine storm in COVID-19. J Crit Intensive Care. 2020;11(Suppl. 1):36-40.

18 Ronco C, Bagshaw SM, Bellomo R, Clark WR, Husain-Syed F, Kellum JA, et al. Extracorporeal blood purification and organ support in the critically ill patient during COVID-19 pandemic: expert review and recommendation. Blood Purif. 2020 May 26;1-11.

19 Cozzolino M, Magagnoli L, Ciceri P, Conte F, Galassi A. Effects of a medium cut-off (Theranova ${ }^{\circ}$ ) dialyser on haemodialysis patients: a prospective, cross-over study. Clin Kidney J. 2019;1-8. 